\title{
3D Computer Reconstruction of the Airway and the Vascular Systems of the Lung of the Domestic Fowl, Gallus gallus Variant domesticus
}

\author{
John N. Maina ${ }^{1,}$, Yolanda Ramonisi ${ }^{1}$, Reatlegile Mashiteng ${ }^{1}$, Lolo Mokae ${ }^{1}$, Jeremy D. Woodward ${ }^{2}$ \\ ${ }^{1}$ Department of Zoology, University of Johannesburg, Auckland Park, Johannesburg 2006, South Africa. \\ ${ }^{2}$ Division of Medical Biochemistry and Structural Biology, Structural Biology Research Unit, University of Cape Town, Observa- \\ tory 7925, South Africa.
}

How to cite this paper: John N. Maina, Yolanda Ramonisi, Reatlegile Mashiteng, Lolo Mokae, Jeremy D. Woodward. (2021) 3D Computer Reconstruction of the Airway and the Vascular Systems of the Lung of the Domestic Fowl, Gallus gallus Variant domesticus. Journal of Applied Mathematics and Computation, 5(2), 89-104.

DOI: 10.26855/jamc.2021.06.004

Received: April 6, 2021

Accepted: April 30, 2021

Published: May 18, 2021

*Corresponding author: John N. Maina, Department of Zoology, University of Johannesburg, Auckland Park, Johannesburg 2006, South Africa.

Email: jmaina@uj.ac.za

\begin{abstract}
The avian respiratory system (the lung-air sac system) is exceptionally structurally complex and functionally efficient. The capacity of powered (active) flight in birds is largely attributed to these features. Although it has been investigated for a longtime, important questions on the bioengineering of the avian respiratory system still remain unclear and controversial. Among these are basis of the airflow dynamics in the lung, the structure and topographic arrangement of the airwayand the vascular systems and the shapes and sizes of the terminal respiratory units. Here, in attempt to resolve some of the issues, the lung of the domestic fowl, Gallus gallus variant domesticus, was investigated by three-dimensional (3D) serial section computer reconstruction. The bronchial-(airway) and the vascular systems were reconstructed and their morphologies thoroughly assessed to determine their morphologies and spatial relationships. Movies of the reconstructions were prepared and rotated to view the structures from different perspectives. Furthermore, the different parts of the pulmonary vasculature were extracted and reinserted into the reconstructions to determine whether anastomoses existed. The most important findings were: three main branches (= rami) of the pulmonary artery deliver venous (deoxygenated) blood to various parts of the lung; three main branches of the pulmonary vein drain arterial (oxygenated) blood from the lung; the third costal sulcus forms the approximate boundary between the cranialand the caudal blood supply- and drainage regions of the lung and; at least up to the level of the interparabronchial arteries and veins, no anastomoses (interconnections) were observed between the branches of the pulmonary artery and vein.
\end{abstract}

\section{Keywords}

Domestic fowl, Lung, Histological sections, 3D reconstruction, Airways, Blood vessels

\section{Introduction}

"Visualizing the three-dimensional (3D) organization of organs is critical for understanding their structure and function". [1]

Through evolution by natural selection [2], cells, tissues, organs and organ-systems that comprise living entities adaptively develop to optimally drive the homeostatic physiological and biochemical life-supporting processes. After the in- 
vention of the compound light microscope, about four-and-half centuries ago by Zacharias Jansen (1580-1638) [3, 4, 5], for nearly 3 centuries, microscopic study of biological structures was limited to visualization of sectional, i.e., two-dimensional (2D), profiles. In the late $19^{\text {th }}$ century, researchers investigating the three-dimensional (3D) properties employed rather crude tools to examine serial sections after projecting their outlines and generating an image of the object orthogonal to the direction of sectioning [6] or by tracing profiles of the object of interest onto stacks of glass sheets [7-9]. Such techniques were still being used when Sydney Brenner in the early 1970's first applied computers to solve the onerous challenge of preparing and robustly examining serial section reconstructions [10,11]. Modern advances in computing power, software engineering, imaging technology and visualization tools have more recently permitted switch to a variety of 3D reconstruction methodologies that have revolutionized the fields of molecular-, cellular-, tissue-, and organ biology [12-24]. Presently, at least at mathematically, even four-dimensional (4D) conceptualization of objects is possible [23, 25-27]. As proposed by Alan Turing [28] in his pioneering work, in biology, function emanates from accretion of various infinitesimal physical and biochemical events that happen between and among the individual structural components at different levels of organization, from molecular-, cellular- to organ-system levels. Thorough understanding of the structure and arrangement of the constitutive components should allow better understanding of the genesis of states and conditions such as physiological processes and events and disease- and pathological states.

Three dimensionality is an ubiquitous property of natural structures [29-31]. The configuration is quintessential to life as we know it. For example, structures that perform functions such as transportation, distribution and exchange of materials and substances such as the bronchial system of the lung that transports air, the gastrointestinal system that conveys food and blood vessels that transfers blood have to develop as tubules and not as sheets or layers such as the peritonealand the meningeal membranes that separate spaces in the body [32, 33]. Experimentally, in cell culture assays, it has been shown that 3D preparations best reproduce the in vivo (natural) conditions and states more suitably compared with the conventional 2D ones [27, 34-43]: cells in 2D cell cultures lose their tissue distinctive properties [39, 44] as differences in mechanical- and biochemical signals disrupt cell-to-cell or cell-to-matrix connections. In drug delivery studies, it has been shown that while many preparations may show success in 2D cell cultures, in 3D environments (e.g. in the body or in 3D culture), large number of cells form masses that limit the efficiency of drug transfer to individual cells [41]. Moreover, in 2D cell cultures, the cells develop a spheroid shape and the clusters form large masses instead of clusters developing from division of single cells [37]: the assemblages are significantly larger than those formed by 3D culture.

Three-dimensional serial section reconstruction is an arduous but highly instructive research technique [19, 45-49]. It entails preparation of spatial models of structures from an adequate number of 2D images [50-52]. However, because of the long time it takes to execute it and the quantity of materials (e.g. number of sections) required to accomplish it and the specialized computational skills needed to perform it well, 3D reconstruction is only rarely employed in morphological studies. The 2D images needed to construct 3D shape can be obtained after preparation of serial sections or by tomographic methods using any measureable signals that can penetrate the specimen such as light, ultrasound, gamma rays, X-rays, neutrons or electrons [19, 53-57]. Dating back to the late $19^{\text {th }}$ century [58-60], use of serial sections for 3D reconstruction is the earliest and longest practiced method by which 3D (spatial) micro-morphological data have been obtained. Although alternative more recent techniques that are faster, cheaper and easier to use and which work in an automated manner are now available, serial histological section 3D reconstruction (SHS-3D-R) still remains an important technique. This is fundamentally because the reconstructions that are generated have better contrast and signal-to-noise ratio. Furthermore, dyes and markers can be applied to well-distinguish and better detect the structural components by coloured or fluorescent signals. SHS-3-R is effective from $\sim 0.2 \mu \mathrm{m}$ resolution and large volumes (in the $1,000^{3}$ voxel range) can be sampled. These features make SHS-3D-R highly effective for preparation of proper, high-resolution 3D micro-morphological images. Recently, advances in optical (light) microscopical sectioning techniques as well as automatic block-face image capturing methods like episcopic fluorescence image capturing (EFIC) or surface imaging microscopy (SIM) [54, 61-68] have profoundly advanced the efficiency and accuracy of capturing images by serial sectioning. Regarding possible sub-micron resolution, compared to the modern 3D reconstruction techniques, conventional light microscopical (histological) sections have certain advantages [55, 69, 70]: the foremost ones are the high contrast and the large sample size that can be investigated [13, 19, 69-72]. Only a few ultramodern 3D reconstruction techniques, e.g., synchrotron-based microcomputer tomography $(\mu \mathrm{CT}$ ) or focused ion beam scanning electron microscopy (FIB/SEM) tomography rival it in the imaging resolution obtained on histological sections $[55,70]$. As more robust methods of applying 3D reconstruction technology continue to be developed and improved, 3D imaging and animation will form a pivotal research method in the morphologist's toolbox. Earlier studies that applied different methods of investigation will need to be re-evaluated. 3D reconstruction has lately found important application in medical fields such as tissue engineering and regenerative medicine [71-74]. Recently, Geyer and Weninger [68] observed that 'imaging plays a central role in all areas of modern science and is one of the most expanding fields of biomedical research'.

To contribute to understanding of the functional design of the avian (bird) lung, here, we have performed 3D histolog- 
ical section reconstruction of the airway- and the vascular (pulmonary artery and vein) systems using multi-view image acquisition of microscopic samples combined with pre- and post-processing steps, including correlation-based image registration, filtering and a combination of manual and automated segmentation. Among the extant air-breathing vertebrates, the avian respiratory system (the lung-air sac system) is structurally the most complex and functionally the most efficient [75-84]. Although it has been investigated for nearly five centuries, i.e., since Coitier [85], some aspects of its biology remain unclear and controversial [83]. Like for other invaginated vertebrate gas exchangers [86], while the respiratory system of birds is on the whole ventilated tidally (i.e., in-and-out), simultaneously, the paleopulmonic part of the lung is ventilated unidirectionally and continuously in a caudocranial direction by synchronized bellows-like actions of the air sacs while the neopulmonic one is ventilated tidally [78, 87]. In the avian lung, the path taken the inspired air is controlled aerodynamically $[88,89]$ and not by anatomical valves or sphincters as was once envisaged. When animals (mammals and birds) of similar body mass are compared, for birds, the avian lung is relatively smaller, has a thinner blood-gas barrier, larger respiratory surface area and larger capillary blood volume, structural features that provide higher anatomical pulmonary diffusing capacity of oxygen [81, 83, 86, 90, 91]. The morphological specializations of the avian lung explain why birds are among the few extant animal taxa that have achieved powered (active) flight, an exceptionally energetically greatly costly form of locomotion [92-94]. Some species of birds even fly nonstop over very long distances while others fly under the hypoxic conditions of extreme altitude [94-98].

An account of this work was presented at the $10^{\text {th }}$ International Conference on Computer Methods at Singapore in July 2019.

\section{Materials and methods}

\subsection{Fixation and processing of the lung}

The Animal Ethics Committee of the University of Johannesburg approved the procedures employed in this study (Clearance Number: 2017-06-29/Maina). A mature domestic fowl, Gallus gallus variant domesticus, was killed by intravenous injection with pentobarbitone sodium (Euthanase ${ }^{\circledR}$ ) into the brachial vein at a dosage of $3 \mathrm{~cm}^{3} \cdot \mathrm{kg}^{-1}$. The lungs were then fixed by intratracheal instillation with phosphate buffered $2.5 \%$ glutaraldehyde $\left(350 \mathrm{mOsm} \mathrm{L} \mathrm{L}^{-1}, \mathrm{pH} 7.4\right)$ at a pressure head of $3 \mathrm{kPa}$. The trachea was ligated and the fixative left in situ for six hours before the lungs were removed from their costovertebral attachments, processed whole by the standard histological procedures and embedded in paraffin wax.

\subsection{Serial sectioning and imaging}

The procedures adopted in preparation of materials and images as well as the techniques applied for 3D reconstruction are shown on Figure 1.

Two-thousand six hundred and eighty-nine $(2,689)$ transverse serial sections were cut at a thickness of $8 \mu \mathrm{m}$, stained with hematoxylin-eosin (H\&E) and mounted in sequence onto glass slides. The complete set of sections comprised the entire lung volume. Thirty seven (37) sections were lost or damaged during sectioning and mounting of sections onto glass slides. In most instances, the lost sections were not consecutive but in one case nine sections spanned a gap of 72 $\mu \mathrm{m}$. An area of $\sim 12.88 \times 9.655 \mathrm{~mm}^{2}$ that included the complete transverse section of the lung was photographed by an Axioskop image analyser (Zeiss Instruments) at a magnification of x10 in uncompressed Tiff image format at a resolution of 2,576 × 1,931 pixels with a calibrated sampling of $5 \mu \mathrm{m} /$ pixel.

\subsection{Image alignment}

Every fifth image was picked and where a section or sections was/were lost or exhibited defects such as folds, tears, compression or insufficient staining, the previous- or the following section was taken. In total, five hundred and thirty five (535) sections representing $40 \mu \mathrm{m}$ in the Z-direction were picked and picked and photographed. The images were manually corrected for brightness and contrast using ImageJ Version 1.4.0. [99] and imported into Spider V.15 [100]. The images were normalised to a mean of 0 and standard deviation of 1 and down-sampled by a factor of 8 using bicubic interpolation to yield a sampling of $40 \mu \mathrm{m}$ in $\mathrm{X}$ and $\mathrm{Y}$. The images were then multiplied by a mask function representing a Gaussian falloff and aligned to one another by maximising the cross-correlation function in $\mathrm{X}, \mathrm{Y}$ and in-plane rotation [14]. The resulting alignment was adequate for resolving the parabronchi and the blood vessels.

\subsection{Segmentation of the reconstruction}

To recognize and segment the air-conducting elements for the reconstruction, an automated method which entailed a Gaussian filter and a threshold was employed [13, 14, 101]. In certain instances where adjacent parabronchi were separated by interparabronchial septa, a borderline was manually drawn around the periphery of the lung. Furthermore, where 
two air-conducting elements were located next to each other, to ensure that they were distinguishable, lines were manually drawn between them. The process was repeated until a satisfactory correspondence between the unprocessed images and the segmented ones [102, 103] was achieved (Figure 2). Blood vessel segmentation was done by manually defining the border of each blood vessel in the original (unprocessed) colour images. These images were then down-sampled and aligned to one another by applying the alignment parameters that were previous determined [13, 14, 101]. To ensure that blood vessels were not mistaken for the air-conducting elements (particularly those lacking blood and erythrocytes in their lumina), the segmented blood vessels were separated from the air-conducting elements.

\subsection{Reconstruction, processing and display}

The above procedure generated three image stacks: ‘air', 'blood' and 'original images' that were changed into volumes using Spider V.15 [100] and low-pass Fourier filtered to a resolution of $160 \mu \mathrm{m}$ with a Gaussian falloff [103]. All three reconstructions were concurrently displayed in UCSF Chimera 1.12 [104], the proper surface threshold value being determined by comparison with the 'original images' volume. Larger blood vessels and airways were visualised by employing an additional low-pass Fourier filter to a resolution of $0.8 \mathrm{~mm}$ and adjusting the threshold value. The segmentation function in Chimera was applied to further segment the blood vessels into arteries and veins (Figure 2).

\section{Results}

The morphologies of the airway- and the vascular (pulmonary artery and vein) systems of the lung of the domestic fowl, Gallus gallus variant domesticus are shown on figures 3-6.

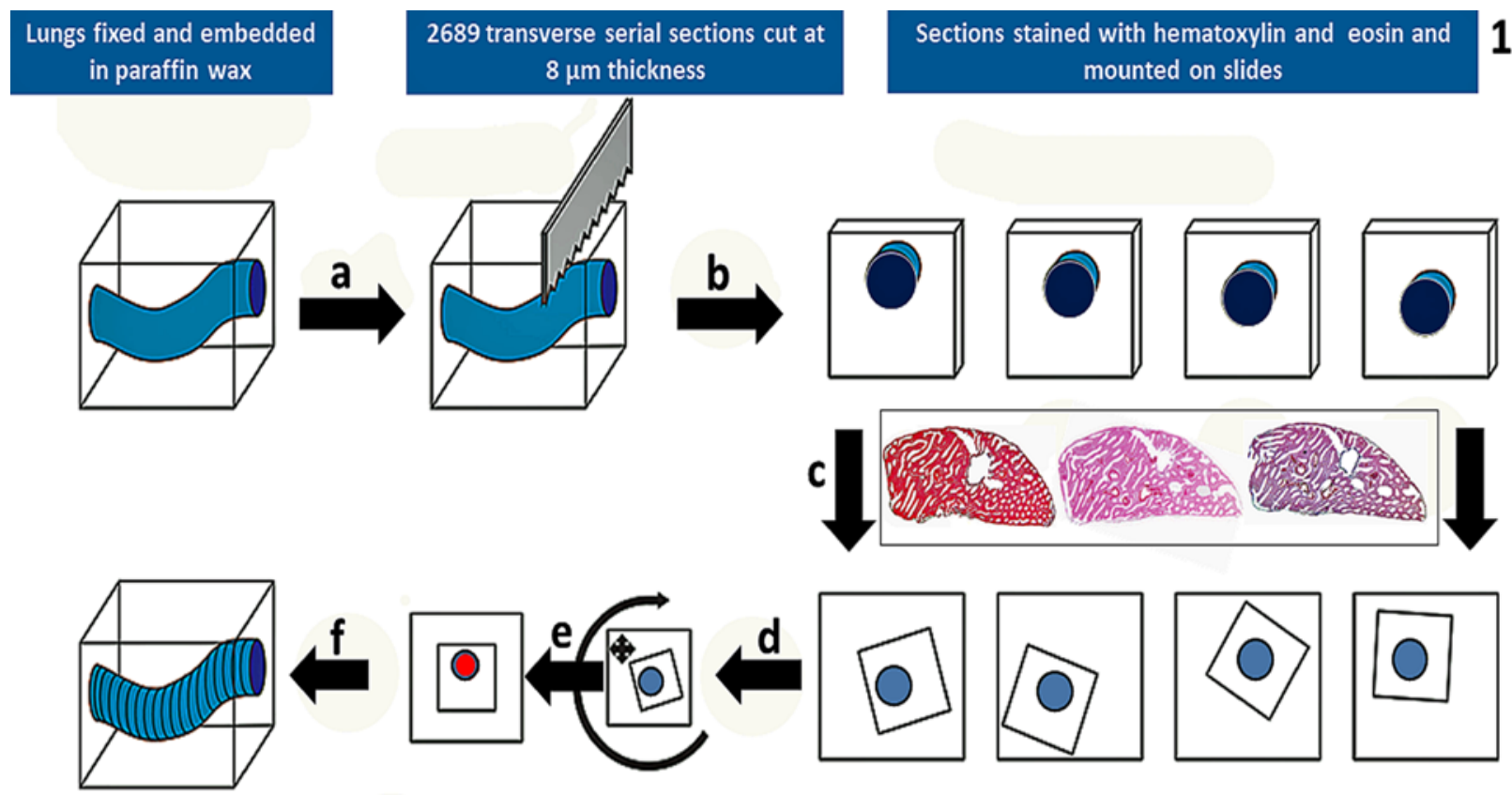

Sections photographed and images digitised
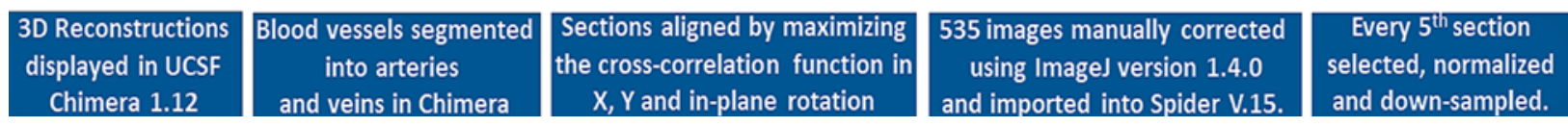

Figure 1. Processes and procedures used in the three-dimensional (3D) reconstruction of the airway- and the vascular systems of the lung of the domestic fowl, Gallus gallus variant domesticus. After processing and blocking the tissues, sectioning was done $8 \mu \mathrm{m}$ thickness and all sections stained; every $5^{\text {th }}$ section was picked and photographed and images normalised and down-sampled; the images were multiplied by a mask function to prevent the image border and the dust particles from contributing to the alignment score; with the previously aligned image (' $n$ ') serving as a template, the images were correlated with one another; for alignment, the image was rotated and translated relative to the previous one until a maximum correlation score was obtained; the newly aligned image was then used as a template to align the next image (' $n+1$ ') and; the process was repeated for the remaining sampled sections. The 3D was done using the UCSF Chimera Package from the Resource for Biocomputing, Visualization and Bioinformatics at the University of California, San Francisco [104]. Further technical details are given in $[13,14,101]$. 


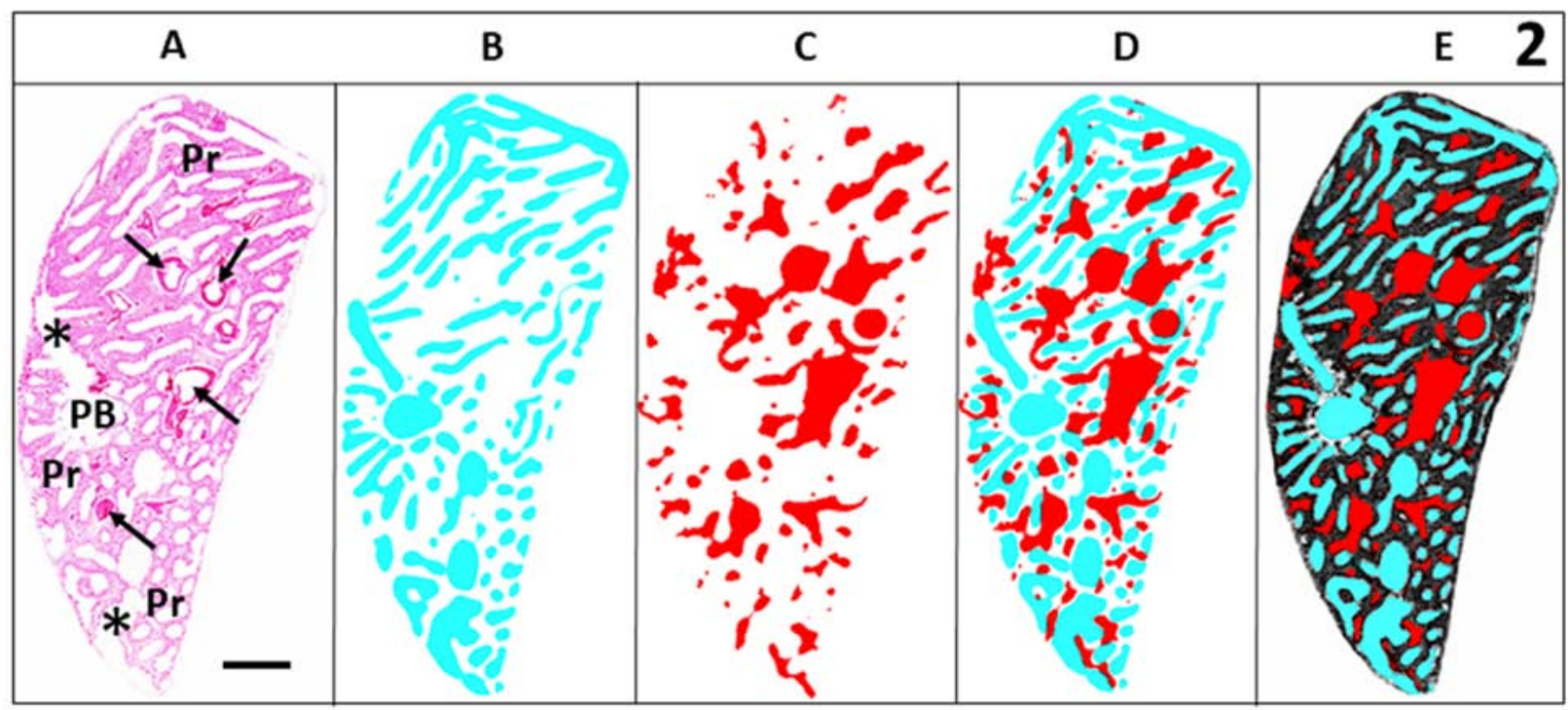

Figure 2. Sampled section (number 525) showing the process of segmentation. Hematoxylin and eosin stained section showing various structural components (A). PB, primary bronchus; asterisks, secondary bronchi; arrows, blood vessels; Pr, parabronchi. The same resampled, normalised and aligned section showing the structural components of the airways (cyan) that are well-resolved (B). Blood vessel- (red) (C) and airway (cyan) (D) segmentation. Excellent match between the structures identifiable on the stained section (A) was obtained (E). Scale bar, $1 \mathrm{~cm}$.

\subsection{Bronchial (airway) system}

The left- and the right extrapulmonary primary bronchi enter the lung at the hilum to form the intrapulmonary primary bronchi (IPPB) or mesobronchi that pass through the lung, changing in shape, size (diameter) and location before exiting on the caudal edge of the lung to deliver air to the caudal air sacs (Figs. 3a-c, e, f; 4; 5a, b, i, j). Figure 4 shows different views of the reconstruction after rotation across various angles. On Figure 5, the arrangement of the airway- and the vascular systems are shown with a transverse section of the lung in the background to show the topographic relationships between the reconstructions. As the IPPB transits the lung, it gives rise to different groups of secondary bronchi on various aspects along its luminal surface (Figs. 3a, c-f; $4 ; 5 \mathrm{a}, \mathrm{b}, \mathrm{i}, \mathrm{j}$ ). Tertiary bronchi or parabronchi interconnect the secondary bronchi (Figure 6): those that join the medioventral secondary bronchi (MVSB) to the mediodorsal secondary bronchi (MDSB) comprise the paleopulmo or the so-called 'old lung' while those that link the MDSB, the lateroventral-, the laterodorsal- and the MVSB form the neopulmo or the so-called 'new lung' [75]. The paleopulmonic parabronchi are arranged as stacks of parallel passageways that are mainly found on the dorsolateral aspect of the lung while the neopulmonic ones are found on the medioventral aspect of the lung (Figure 6). The paleopulmonic parabronchi are larger in size (diameter and length) and anastomose regularly while the neopulmonic ones are smaller in size and interconnect profusely (Figure 6).

\subsection{Pulmonary vein (PV)}

On entering the lung at the hilum just ventral to the origin of the first MVSB, three main branches or rami connect to form the PV (Figs. 3b, d, e, f; 4): these are the accessory-, the cranial- and the caudomedial branches. The PV drains arterial (oxygenated) blood from different parts of the lung and return it to the heart for circulation to the rest of the body. The accessory branch which is the last branch to join the other branches to form the PV drains blood from a smaller part of the lung ventral to hilum; the cranial branch drains blood from the craniodorsal region of the lung immediately before the third costal sulcus and the caudomedial branch (which is the most direct continuation of the PV collects blood from most of the lung caudal to the third costal sulcus (Figs. 3b, d-f; 4; 5c, d, g-j). The three rami of the PV essentially divide the lung into roughly a cranial- and a caudal drainage areas along a transverse plane that passes between the second- and third costal sulcus (Figs. 3c-f; 5c, d, g-j), with the cranial part of the lung drained by the accessory- and the cranial branches and the caudal part by the caudomedial branch. Along the median longitudinal plane that is more or less marked by the course followed by the IPPB, the lateral aspect of the caudal part of the lung is drained by a smaller caudolateral branch of the main caudomedial branch (Fig. 5d, h, j). Up to the level of the interparabronchial veins, no connections 
(anastomoses) were observed between the branches of the PV and the PA.

\subsection{Pulmonary artery (PA)}

At the hilum, the PA is separated from the PV by the primary bronchus (Figs. 3e; 4; 5g, h, i, j). Delivering venous (deoxygenated) blood to the lung, the PA branches into craniomedial-, caudomedial- and accessory branches close to the hilum (Figs. 3c-f; 5e, f-j): it lies lateral to the PV (Fig. 3d-f). In this study, the hilal region was not included in the reconstruction as it was inadvertently removed while clearing the tissues adhering on the surface of the lung. The largest part of the craniodorsal part of the lung is supplied with venous blood by the cranial branch of the PA (Figs. 3c-f, 5e-j); the caudomedial branch supplies blood to the part of the lung caudal to a transverse plane passing along the third costal sulcus and gives off as many as four small branches that radiate out towards the dorsal and ventral parts of the lung and; the accessory branch delivers blood to the ventral part of the lung (Figs. 3c-f; 4; 5e-j). Up to the level of the interparabronchial arteries, no connections (anastomoses) were observed between the branches of the PA and the PV.

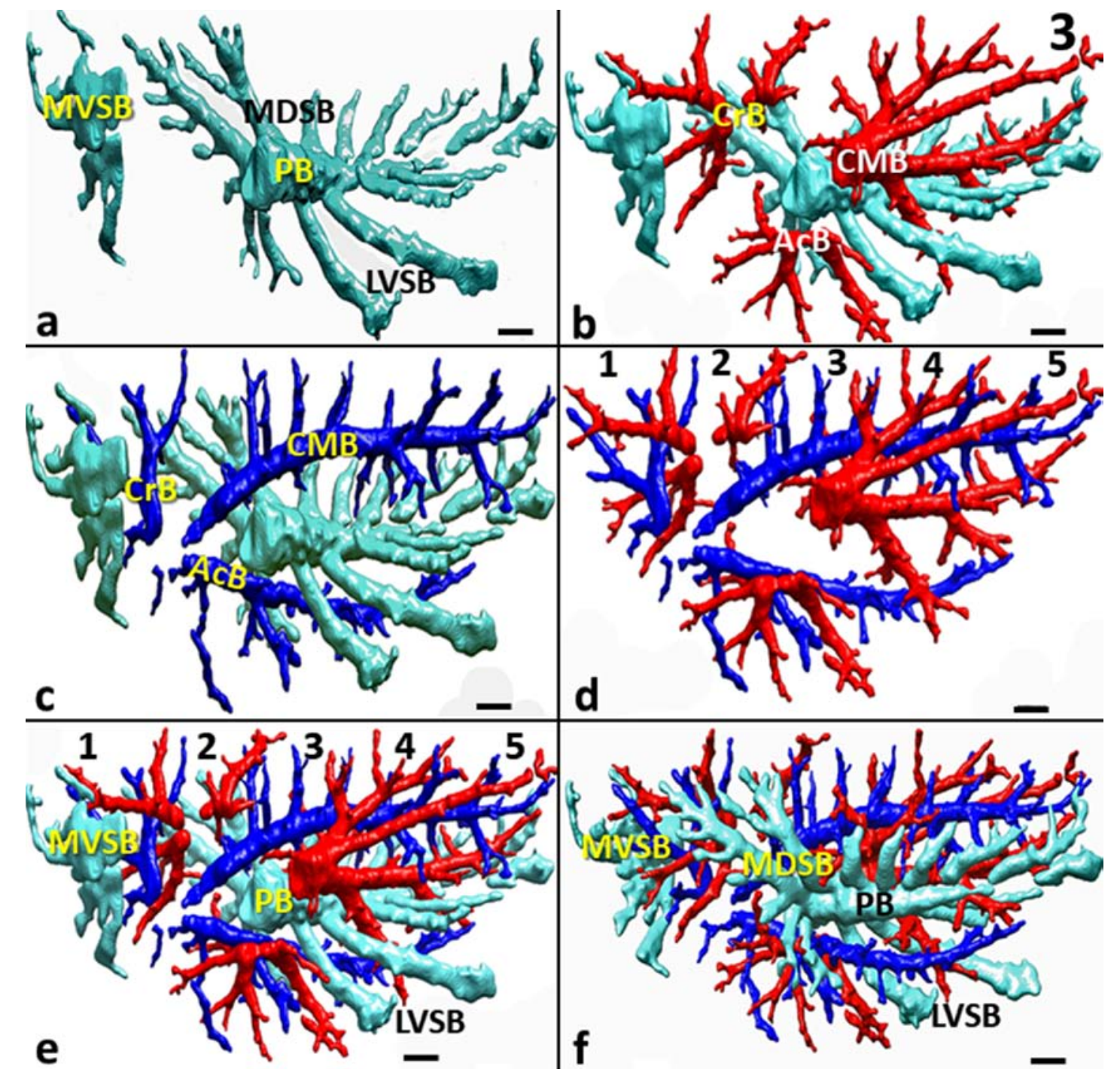

Figure 3. Three-dimensional (3D) computer reconstruction of the airway- and vascular systems of the lung of the domestic fowl, Gallus gallus variant domesticus that are computationally cropped to well-show the structures: medial- (a-e) and lateral (f) views. The airways are shown in cyan, the pulmonary artery in blue and the pulmonary vein in red. The numbering 1-5 (Figs. d, e) shows costal sulci (impressions made by the ribs) in a craniocaudal direction. Three main branches, namely the accessory(AcB), the cranial- (CrB) and caudal medial (CMB) branches form the pulmonary vein and the pulmonary artery comprises similar braches, namely the accessory- (AcB), the cranial- (CrB) and caudal medial (CMB) branches. PB, primary bronchus; MVSB, medioventral secondary bronchi; MDSB, mediodorsal secondary bronchi; LVSB, lateroventral secondary bronchi. Scale bar, $0.5 \mathrm{~cm}$. 

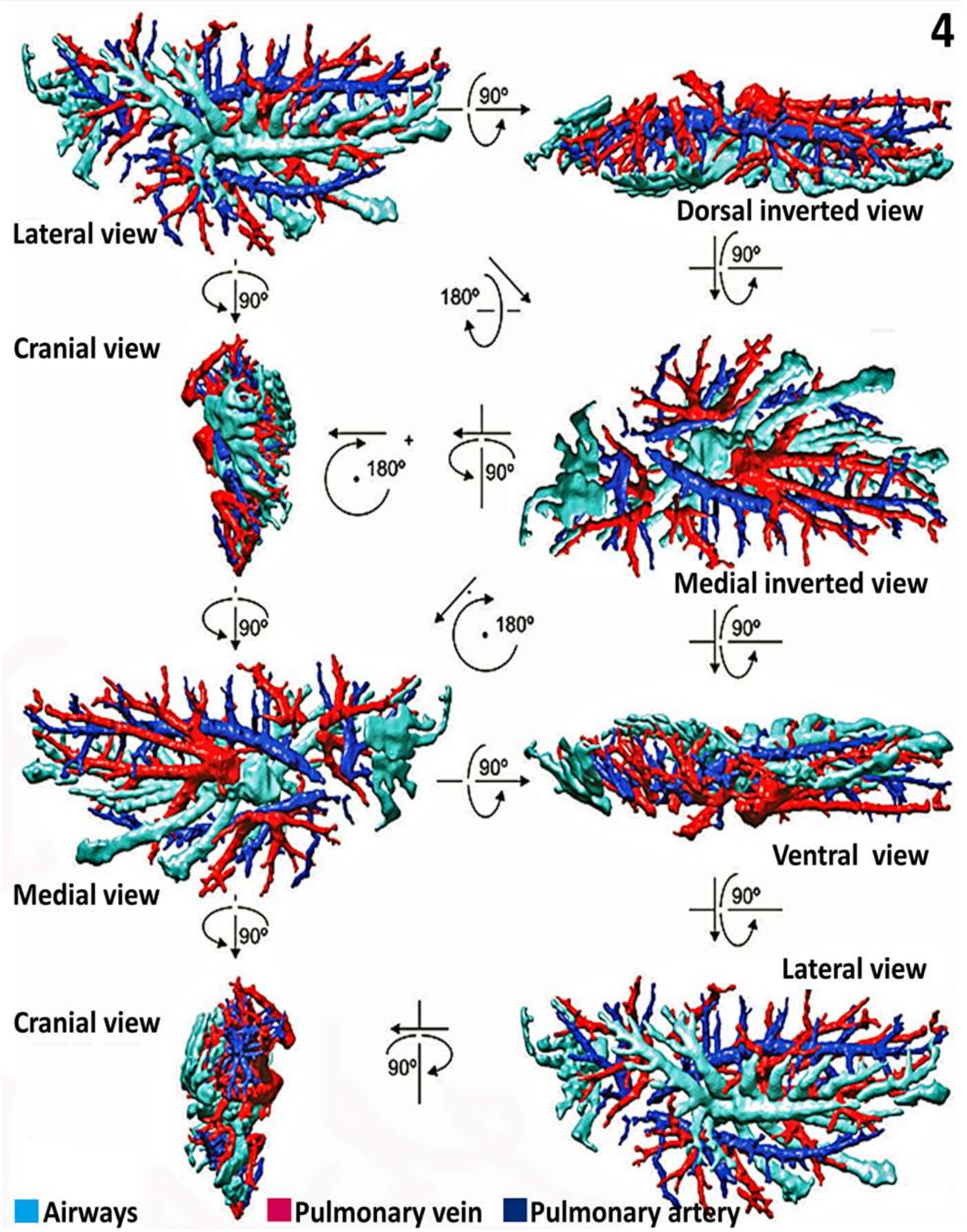

Figure 4. Different views of the reconstructions of the airway- and the vascular systems of the lung of the domestic fowl, Gallus gallus variant domesticus, shown on Figure 3. 


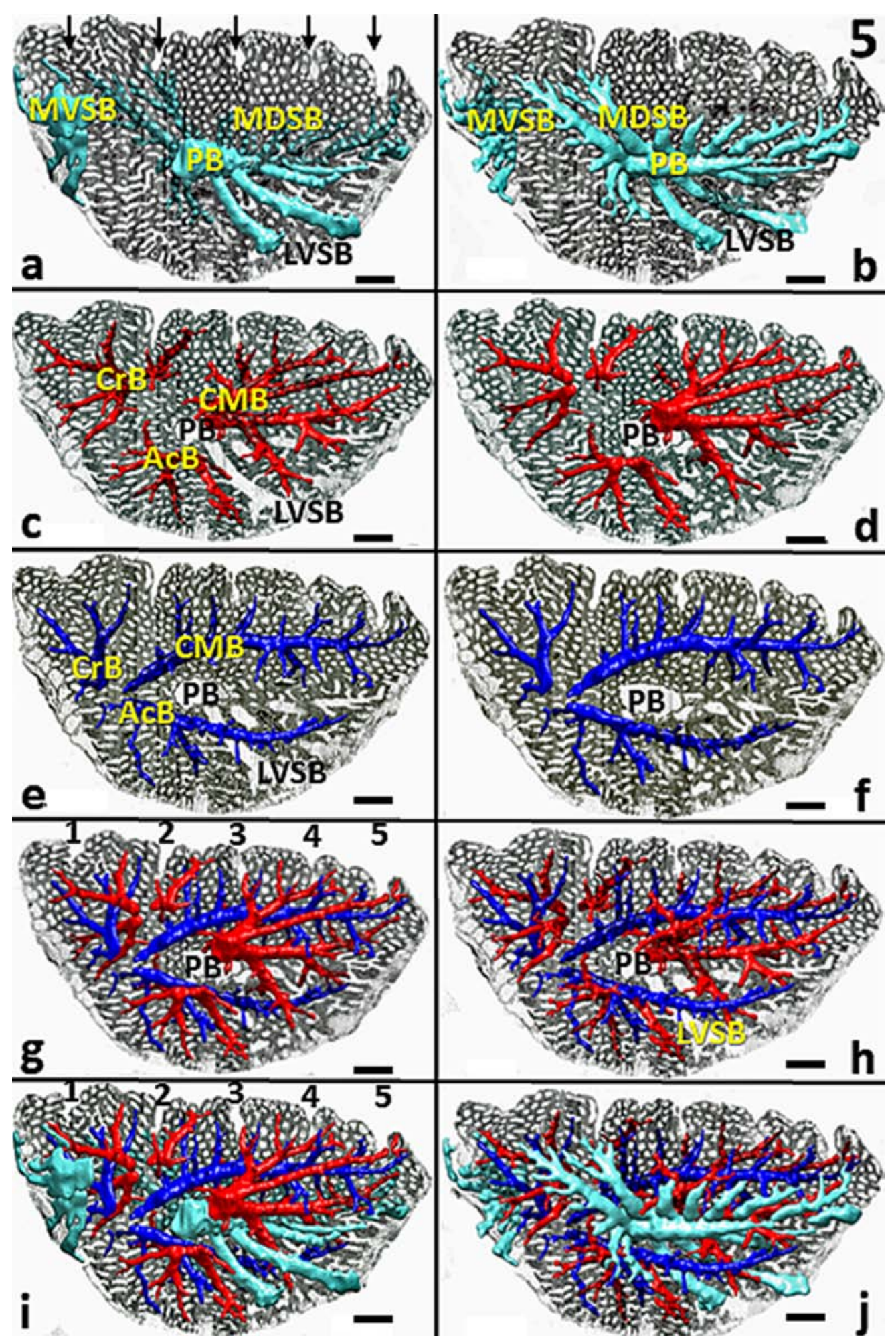

Figure 5. Medial and lateral views of the three-dimensional (3D) reconstructions of the airways (bronchi) shown in cyan (Fig. $4 a, b, i, j)$ and blood vessels, i.e., pulmonary vein (red) (Fig. 4c, d, g-j) and pulmonary artery (blue) (Fig. 4e-j). The reconstructions are overlaid on a cross-section of the lung to show the locations and topographical relationships of the airway- and vascular structures. The numbers 1-5 [shown with arrows on figure 4a and in consecutive numbers (1-5) on figure 4g, $i$ are the costal sulci, i.e., the markings made by the ribs on the surface of the lung]. PB, primary bronchus; MVSB, medioventral secondary bronchi; MDSB, mediodorsal secondary bronchi; LVSB, lateroventral secondary bronchi. Scale bar, $0.5 \mathrm{~cm}$. 


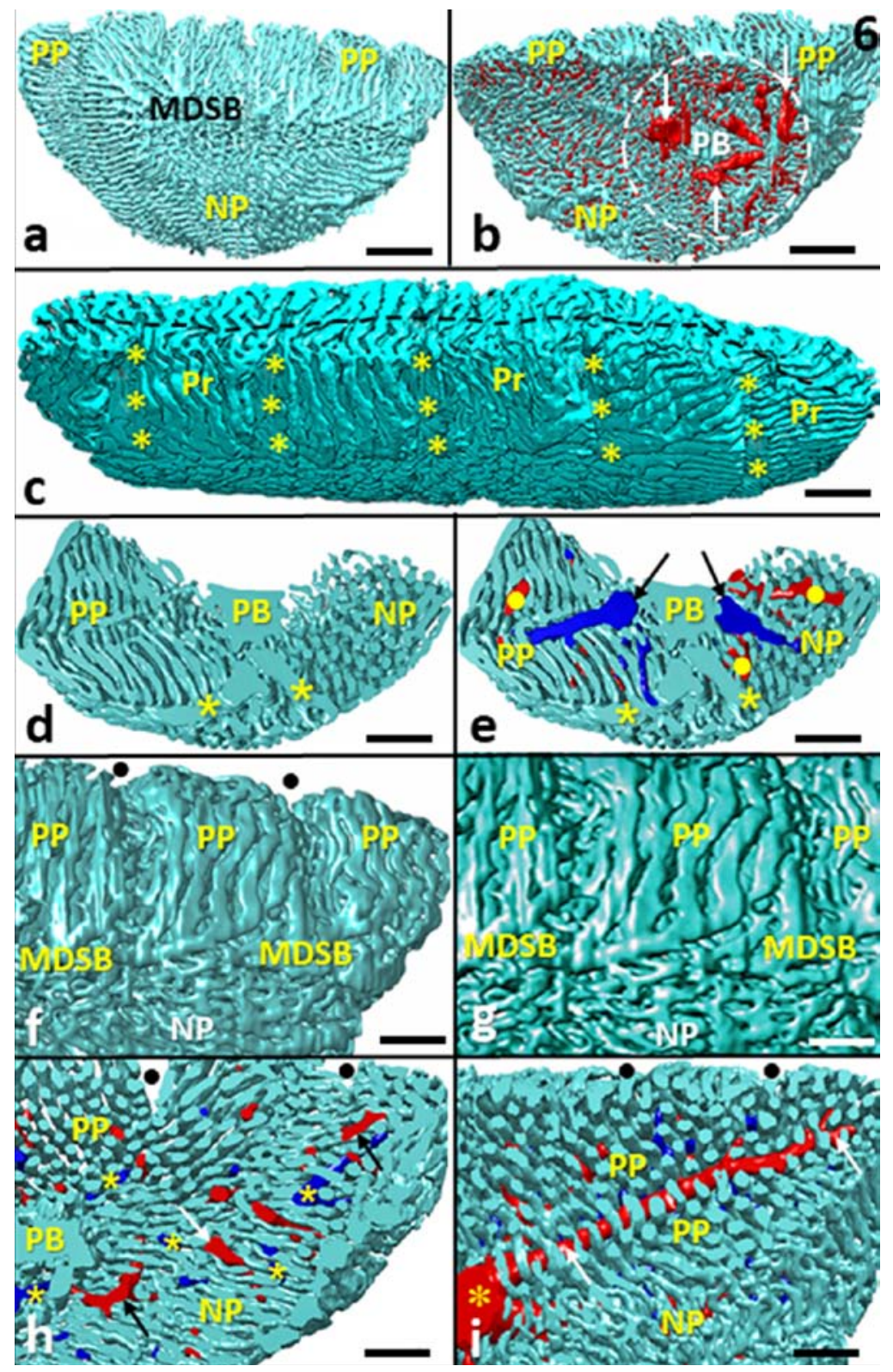

Figure 6. Three-dimensional (3D) reconstructions of the airway (bronchial) system of the lung of the domestic fowl, Gallus gallus variant domesticus. Fig. 5a is a lateral view and Fig. $5 \mathrm{~b}$ medial one showing the paleopulmonic- (PP) and the neopulmonic (NP) regions of the lung. MDSB, mediodorsal secondary bronchi. Fig. 5b) shows the hilal region of the lung (dashed circle), the primary bronchus (PB) and branches of the pulmonary vein (arrows). Fig. 5c is a dorsolateral view of the reconstructed lung showing paleopulmonic parabronchi (Pr) from the mediodorsal secondary bronchi connecting with those from the medioventral secondary bronchi (MVSB) (located on the medial aspect of the lung) and joining at the linea anastomotica or the line of anastomosis (dashed line). The costal sulci which are the markings left by the ribs on the lung are shown by stars. Fig. 5, d, e: Transverse section of the reconstructed lung showing primary bronchus (PB), paleopulmonic- (PP) and neopulmonic (NP) regions of the lung. Stars, secondary bronchi; arrows, branches of the pulmonary artery; dots, branches of the pulmonary vein. Fig. 5f, g: Close ups of the lateral aspects of the reconstructed lung showing paleopulmonic- (PP) (originating from MDSB) and neopulmonic (NP) parabronchi of the lung: the former are larger in diameter and are arranged in stacks while the later are relatively smaller and anastomose profusely. Fig. $5 \mathrm{~h}$, i: Longitudinal section of the lung showing paleopulmonic parabronchi (PP) (cut transversely) that are arranged in stacks and neopulmonic parabronchi (NP) that anastomose profusely. On Fig. 5h, the branches of the pulmonary vein are shown by arrows and those of the pulmonary artery by arrows.

$\mathrm{PB}$, primary bronchus; dots, costal sulci. On Fig. $5 \mathrm{i}$, a branch of the pulmonary vein (star) is seen passing across paleopulmonic parabronchi (PP) (arrows). NP, neopulmo. Scale bars: a, b, 1 cm; c, 0.5 cm; d, e, 1 cm; f, 0.5 mm; g, 0.2 mm; h, 0.5 mm; i, $0.2 \mathrm{~mm}$. 


\section{Discussion}

The structure and function of the avian lung have been investigated by various techniques that have among others comprised injection of markers such as microspheres into the blood vessels and following the paths they have taken microscopically, casting the airways and the blood vessels with materials such as microfilm, silicone, mercox and latex rubber and by different types of instruments such as confocal- and transmission- and the scanning electron microscopes [75-81, 84, 86-88, 90, 91, 105-118]. While highlighting the notable structural features that were observed in this study, previous observations are corroborated and the robusticity of the technique of 3D computational reconstruction as means of investigating the morphologies of complex biological structures is underscored. The shapes, the connections and the topographic relationships of the structural components that comprise the avian lung are unclear and contentious [83, 115, 116] and where interspecific differences occur, it is uncertain whether the variations have originated from developmental processes or have emanated from the environmental conditions under which eggs were incubated and hatched and the lifestyles that birds pursued on maturity.

As observed by Abdalla [105] and Abdalla and King [106, 107] and corroborated in this study, compared with the mammalian lung where the pulmonary artery closely tracks and patterns the airways and the vein located intersegmentally [119], for the avian lung, such close relationship does not exist between the airway- and the vascular systems. The morphological differences between the mammalian- [120] and the avian [121] lungs may explain the more complex arrangement of the structures of the avian lung and its relatively greater respiratory efficiency [79, 102, 122-124]. In more derived species of birds, e.g., the songbirds or passeriforms, the lung comprises two structurally and functionally distinctive parts, namely the relatively larger paleopulmo (old lung) that develops before the smaller neopulmo (new lung) [75, 122-124]: the paleopulmo is largely located on the dorsal aspect of the lung and is ventilated continuously and unidirectionally in a caudocranial direction by synchronised actions of the air sacs while the neopulmo is mainly found on the ventral aspect and is ventilated tidally [75, 78, 87]. While the paleopulmo exists in all adult avian lungs [75, 78, 79], in less derived birds such as the kiwi (Genus: Apteryx), the neopulmo is lacking [79]. As corroborated in this study, the paleopulmonic parabronchi are arranged in parallel stacks that anastomose periodically while those of the neopulmo anastomose more profusely. The topographic and the morphologic differences between the airways and the blood vessels of the avian lung (compared to the mammalian one) may arise from the fact that the two parts of the lung (paleopulmo and neopulmo) develop at different times in different locations [123, 124]. In the nascent lung bud of the mammalian- and the avian lungs, the mesenchymal cells that form the airways and the blood vessels originate at the hilum and extend outwards [119, 120, 123-126].

Regarding the studies by Abdalla [105] and Abdalla and King [106, 107] that entailed detailed study of the morphologies of the pulmonary vasculature of the domestic fowl, Gallus gallus variant domesticus by microsphere injection followed by microscopic follow ups, while corroborating most of the observations made by the investigators, here, 3D computer recostruction revealed certain variations. While main branches of the PA, namely the accesory-, the caudolateral-, the caudomedial- and the cranial branches were reported by Abdalla [105] and Abdalla and King [106, 107], we observed only three main branches (= rami); for the PV, we observed three main branches while Abdalla [105] and Abdalla and King [106, 107] reported two branches, namely a cranial- and a caudal branch. Both in Abadalla [105] and in this study, the third costal sulcus formed the approximate boundary between the cranial- and the caudal blood supply/drainage regions of the lung occur and at least up to the level of the interparabronchial arteries and veins, no anastomoses (interconnections) were found between the branches of the PA and the PV. Because of the capability of rotating the reconstructions and extracting and repositioning the various parts of the reconstructions, it can be confirmed that arterial-venous anastomoses do not occur between the vascular systems of the avian lung ahead of the interparabronchial arteries and veins of at least that of the domestic fowl. It suggests that in such lungs, the air flowing along the parabronchial lumina and the venous blood flowing in the parabronchial exchange tissue do not equilibrate and that gas exchange should occur all along the parabronchial exchange tissue. Potentially, such a design forms a highly efficient gas exchange system with functional reserve.

\section{Conclusion}

Compared with other techniques that have hitherto been employed to study the morphologies of the airway- and the vascular systems of the avian lung, 3D computer reconstruction is highly instructive. Here, movies of the reconstructions and ability to extract and reposition the various structures allowed for thorough assessment of the preparations. As highlighted by Baghaie et al. [21], contrary to general assumption, the scanning electron microscope (SEM), an instrument commonly used to view surfaces of biological structures at micro-scale does not provide 3D images. The visuals are essentially 2D since they lack a measure of true depth. Furthermore, although the preparations can to an extent be mechanically rotated for the surface to be viewed from different angles, the mounted side cannot be accessed. However, recently, 
several SEM techniques that can be used to obtain 3D details of a biological sample have been developed [127-129]. Some of the techniques can be performed on most microscopes while others require specialized instrumentation and software.

\section{Acknowledgement}

This work was funded by the National Research Foundation (NRF) of South Africa [Grant number: KIC $180412319872(115503)]$.

\section{References}

[1] Sergé, A., Bailly, A. L., Aurrand-Lions, M., Imhof, B. A., Irla, M. (2015). For 3D: Full organ reconstruction in 3D, an automatized tool for deciphering the complexity of lymphoid organs. J Immunol Methods, 2015, 424: 32-42.

[2] Darwin, C. (1859). On the origin of species by means of natural selection, or the preservation of favoured races in the struggle for life. London: John Murry.

[3] Nachtigall, W. (1997). Exploring with the microscope: a book of discovery and learning. London: Sterling Publications.

[4] Savile, B., Bracegirdle, B. (1998). Introduction to light microscopy. New York: Springer.

[5] Van Helden, A., Dupré, S., Rob, V. G., Zuidervaart, H. (eds). (2011). The origins of the telescope. Amsterdam: The Amsterdam University Press.

[6] His, W. (1880). Anatomie menschlicher Embryonen. Leipzig: Vogel.

[7] Kastschenko, N. (1886). Methode zur genauen rekonstruktion kleinerer makroskopischer gegenstande. Arch Anat Physiologie Abteilung ,1886; 1: 388-394.

[8] Odhner, T. (1911). Zum naturlichen System der digenen Trematoden IV. Zool Anz, 1911; 38: 513-531.

[9] Ohta, Y., Millhouse, E. W. (1967). Glass plate reconstruction from serial sections used in the study of neonatal biliary atresia. In: Stereology (Elias H ed). Berlin: Springer; 1967: pp. 302-303.

[10] Ward, S., Thomson, N., White, J. G., Brenner S. (1975). Electron microscopical reconstruction of the anterior sensory anatomy of the nematode, Caenorhabditis elegans. J Comp Neuro, 1975; 160: 313-337.

[11] Brenner, S. (2009). In the beginning was the worm. Genetics, 2009; 182: 413-415.

[12] Teutsch, H. F., Schuerfeld, D., Groezinger, E. (1999). Three-dimensional reconstruction of parenchymal units in the liver of the rat. Hepatology, 1999; 29: 494-505.

[13] Woodward, J. D., Maina, J. N. (2005). A 3D digital reconstruction of the components of the gas exchange tissue of the lung of the muscovy duck, Cairina moschata. J Anat, 2005; 206: 477-492.

[14] Woodward, J. D., Maina, J. N. (2008). Study of the structure of the air and blood capillaries of the gas exchange tissue of the avian lung by serial section three-dimensional reconstruction. J Microsc, 2008; 230: 84-93.

[15] Song, W. C., Hu, K. S., Kim, H. J., Koh, K. S. (2007). A study of the secretion mechanism of the sebaceous gland using three-dimensional reconstruction to examine the morphological relationship between the sebaceous gland and the arrector pili muscle in the follicular unit. British J Dermatol, 2007; 157: 325-330.

[16] Sun, K., Zhang, J., Chen, T., Chen, Z., Chen, Z., Li, Z., H., Hu, P. (2009). Three-dimensional reconstruction and visualization of the median nerve from serial tissue sections. Microsurgery, 2009; 29: 573-577.

[17] Penczek, P. A. (2010). Fundamentals of three-dimensional reconstruction from projections. Methods in Enzymol, 2010; 482: 1-33.

[18] Wu, X., Yu, Z., Liu, N. (2012). Comparison of approaches for microscopic imaging of skin lymphatic vessels. Scanning, 2012; 34: 174-180.

[19] Onozato, M. L., Klepeis, V. E., Yagi, Y., Mino-Kenudson, M. (2012). A role of three-dimensional (3D)- reconstruction in the classification of lung adenocarcinoma. Anal Cell Pathol, 2012; 35: 79-84.

[20] Miranda, K., Girard-Dias, W., Attias, M., de Souza, W., Ramos, I. (2015). Three-dimensional reconstruction by electron microscopy in the life sciences: an introduction for cell and tissue biology. Mol Reprod Dev, 2015; 82: 530-547.

[21] Baghaie, A., Tafti, A. P., Owen, H. A., D’Souza, R. M., Yu, Z. (2017). Three-dimensional reconstruction of highly 
complex microscopic samples using scanning electron microscopy and optical flow estimation. PLoS One, 2017; 12(4): e0175078.

[22] Chozinski, T. J., Mao, C., Halpern, A. R., Pippin, J. W., Shankland, S. J., Alpers, C. E., Najafian, R., Vaughan, J. G. Volumetric, nanoscale optical imaging of mouse and human kidney via expansion microscopy. Sci Rep., 2018; 8:10396. (doi 10.1038/s41598-018-28694-2).

[23] Kartasalo, K., Latonen, L., Vihinen, J., Visakorpi1, T., Nykter, M., Ruusuvuoril, P. (2018). Comparative analysis of tissue reconstruction algorithms for 3D histology. Bioinformatics, 2018; 34: 3013-3021.

[24] Oliveira, M., Duarte, S. B., Giacomini, G., Pereira, P. C. M., de Souza, L. R., Miranda, J. R. A., Pina, D. R. A lung image reconstruction from computed radiography images as a tool to tuberculosis treatment control. J Venom Animal Toxins Incl Trop Dis, 2019; 25: e144918. (doi.org/10.1590/1678-9199-jvatitd-a449-19)

[25] Zhou, J. (1991). Visualization of four dimensional space and its applications (PhD Thesis; Purdue University Technical Report); Number 91-084:1991.

[26] Lorenz, U. J., Zewail, A. Observing liquid flow in nanotubes by 4D electron microscopy. Science, 2014; 344: 1496-1500.

[27] Bissell, M. J. (2017). Goodbye flat biology—time for the 3rd and the 4th dimensions. J Cell Sci., 2017; 130: 3-5.

[28] Turing, A. M. (1952). The chemical basis of morphogenesis. Philos Trans R Soc (Lond) B, 1952; 237: 37-72.

[29] French, R. (1988). Invention and evolution design in nature and engineering. Cambridge: Cambridge University Press; 1988.

[30] Sung, W. (2018). Statistical physics of biological matter. Dordrecht: Springer.

[31] Sharp, T. A., Merkel, M., Manning, M. L., Liu, A. J. (2019). Inferring statistical properties of 3D cell geometry from 2D slices. PLoS One, 2019; 14, e0209892. (doi.org/10.1371/journal.pone.0209892)

[32] Murakami, M. (2012). Signaling required for blood vessel maintenance: molecular basis and pathological manifestations. Int J Vasc Med Article 2012. (doi:10.1155/2012/293641)

[33] Simons, M., Gordon, E., Claesson-Welsh L. (2016). Mechanisms and regulation of endothelial VEGF receptor signaling. Nature Rev Mol Cell Biol, 2016; 17(10). (doi: 10.1038/nrm.2016.87)

[34] Lee, G. Y., Keny, P. A., Lee, E. H., Bissell, M. J. (2007). Three-dimensional culture models of normal and malignant breast epithelial cells. Nature Methods, 2007; 4: 359-365.

[35] Glauco, S. (2010). Three-dimensional tissue culture based on magnetic cell levitation. Nature Nanotech, 2010; 5: 291-296.

[36] Baker, B. M., Chen, C. S. (2012). Deconstructing the third dimension—how 3D culture microenvironments alter cellular cues. J Cell Sci., 2012; 125: 3015-3024.

[37] Edmondson, R., Broglie, J., Adcock, A., Yang, L. (2014). Three-dimensional cell culture systems and their applications in drug discovery and cell-based biosensors. Assay Drug Dev Technol, 2014; 12: 207-218.

[38] Zanoni, M., Piccinini, F., Arienti, C., Zamagni, A., Santi, S., Polico, R., Bevilacqua, A., Tesei, A. (2016). 3D tumor spheroid models for in vitro therapeutic screening: a systematic approach to enhance the biological relevance of data obtained. Sci Rep, 2016; 6: 19103. (doi: 10.1038/srep19103)

[39] Powell, K. (2017). Adding depth to cell culture. Sci Technol Feature, 2017; 361; 6402.

[40] Ravi, M., Paramesh, V., Kaviya, S. R., Anuradha, E., Solomon, P. F. D. (2015). 3D cell culture systems: advantages and applications. J Cell Physiol, 2015; 230: 16-26.

[41] Cavo, M., Fato, M., Peñuela, L., Beltrame, F., Raiteri, R., Scaglione, S. (2016). Microenvironment complexity and matrix stiffness regulate breast cancer cell activity in a 3D in vitro model. Sci Rep, 2016; 6: 35367. (doi:10.1038/srep35367)

[42] Fang, Y., Eglen, R. (2017). Three-dimensional cell cultures in drug discovery and development. SLAS Discov: Advancing Life Sci., 2017; 22: 456-472.

[43] Langhans, S. (2018). Three-dimensional in vitro cell culture models in drug discovery and drug repositioning. Front Pharmacol, 2018; 22: 456-472.

[44] Haycock, J. W. (2011). 3D cell culture: a review of current approaches and techniques. Methods Molec Biol, 2011; 695: 1-15. 
[45] Levinthal, C., Ware, R. (1972). Three-dimensional reconstruction from serial sections. Nature, 1972; 236: 207-209.

[46] Perkins, W. J., Green, R. J. (1982). Three-dimensional reconstruction of biological sections. J Biomed Engineer, 1982; 4: 37-43.

[47] Latamore, G. B. (1983). Creating 3-D models for medical research. Comput Graphics World, 1983; 5: 31-38.

[48] Mercer, R. R., Crapo, J. D. Structure of the gas exchange region of the lungs determined by three- dimensional reconstructions. In: Toxicology of the lung (Gardner DE, Crapo JD, Massaro EJ eds). New York; Raven Press: pp. 43-70.

[49] Xu, Y. H., Lahvis, G., Edwards, H., Pilot, H. C. (2004). Three-dimensional reconstruction from serial sections in PC-windows platform by using 3D_viewer. Comput Methods Programs Biomed, 2004; 76: 143-154.

[50] Anderson, J. R., Wilcox, M. J., Wade, P. R., Barrett, S. F. (2003). Segmentation and 3D reconstruction of biological cells from serial slice images. Biomed Sci Instrument, 2003; 39: 117-122.

[51] Rosenhain, S., Magnuska, Z. A., Yamoah, G. G., Rawashdeh, W. A., Kiessling, F., Gremse, F. (2018). A preclinical micro-computed tomography database including 3D whole body organ segmentations. Sci Data 5 2018. (Article number 180294)

[52] Vints, K., Vandael, D., Baatsen, P., Pavie, B., Vernaillen, F., Corthout, N., Rybakin, V., Munck, S., Gounko, N. V. Modernization of Golgi staining techniques for high-resolution, 3-dimensional imaging of individual neurons. Sci Rep., 2019; 9(1). (Article number 130) (doi 10.1038/s41598-018-373777-x)

[53] Herman, G. T. (2009). Fundamentals of computerized tomography: image reconstruction from projection, 2nd edition. Berlin; Springer.

[54] Diaspro, A. E. (ed.) (2001). Confocal and two-photon microscopy: foundations, applications and advances. Wiley; VCH): 2001.

[55] Handschuh, S., Schwaha, T., Metscher, B. D. (2010). Showing their true colors: a practical approach to volume rendering from serial sections. BMC Dev Biol, 2010; 10: 41. (doi 10.1186/1471-213X-10-41)

[56] Wang, C. W., Gosno, E. B., Li, Y. S. (2015). Fully automatic and robust 3D registration of serial-section microscopic images. Sci Rep, 2015; 5: 15051. (doi 10.1038/srep15051)

[57] Wang, Y., Xu, R., Luo, G., Wu, J. (2015). Three-dimensional reconstruction of light microscopy image sections: present and future. Front Med., 2015; 9:30-45.

[58] Born, G. (1883). Die Plattenmodelliermethode. Arch Mikrosk Anat, 1883; 22: 584-599.

[59] Strasser, H. (1886). Ueber das Studium der Schnittserien und über die Hülfsmittel, welche die Reconstruction der zerlegten Form erleichtern. Zeitschrifft Wissen Mikrosk, 1886; 3: 179-195.

[60] Strasser, H. (1987). Ueber die Methoden der plastischen Rekonstruktion. Zeittschrift Wissen Mikrosk, 1987; 4: 168-208.

[61] Weninger, W. J., Mohun, T. (2002). Phenotyping transgenic embryos: a rapid 3-D screening method based on episcopic fluorescence image capturing. Nature Genetics, 2002; 30: 59-65.

[62] Ewald, A. J., McBride, H., Reddington, M., Fraser, S. E., Kerschmann, R. (2002). Surface imaging microscopy, an automated method for visualizing whole embryo samples in three dimensions at high resolution. Dev Dyn, 2002; 225: 369-375.

[63] Rosenthal, J., Mangal, V., Walker, D., Bennett, M., Mohun, T. J., Lo, C. W. (2004). Rapid high resolution three-dimensional reconstruction of embryos with episcopic fluorescence image capture. Birth Defects Res C Embryol Today, 2004; 72: 213-223.

[64] Keller, P. J., Schmidt, A. D., Wittbrodt, J., Stelzer, E. H. (2008). Reconstruction of zebrafish early embryonic development by scanned light sheet microscopy. Science, 2008; 322: 1065-1069.

[65] Tsuchiya, M., Yamada, S. (2014). High-resolution histological 3D-imaging: episcopic fluorescence image capture is widely applied for experimental animals. Congenit Anom (Kyoto), 2014; 54: 250-251.

[66] Takaishi, R., Aoyama, T., Zhang, X., Higuchi, S., Yamada, S., Takakuwa, T. (2014). Three-dimensional reconstruction of rat knee joint using episcopic fluorescence image capture. Osteoarthtitis Cartilage, 2014; 22: 1401-1409.

[67] Kong, W., Du, W., Liu, K., Liu, H., Zhao, Z., Pu, M., Wang, C., Luo, X. (2018). Surface imaging microscopy with turntable penetration depth as short as $20 \mathrm{~nm}$ by employing the hyperbolic metamaterials. J Materials Chem C., 
2018; 6(7). (doi: 10.1039/C7TC04748G)

[68] Geyer, S. H., Weninger, W. J. (2019). High-resolution episcopic microscopy (HREM): looking back on 13 years of successful generation of digital volume data of organic material for 3D visualisation and 3D display. Appl Sci., 2019; 9: 3826. (doi:10.3390/app9183826)

[69] Braumann, U. D., Scherf, N., Einen, J., Horn, L. C., Wentzensen, N., Loeffler, M., Kuska, J. P. (2007). Large histological serial sections for computational tissue volume reconstruction. Methods Inf Med., 2007; 46: 614-622.

[70] Roberts, N., Magee, D., Song, Y., Brabazon, K., Shires, M., Crellin, D., Orsi, N. M., Quirke, R., Quirke, R., Quirke, P., Treanor, D. (2012). Towards routine use of 3D histopathology as a research tool. Am J Pathol., 2012; 180: 1835-1842.

[71] Liu, B., Gao, X. L., Yin, H. X., Luo, S. Q., Lu, J. (2007). A detailed 3D model of the guinea pig cochlea. Brain Struct Funct, 2007; 212: 223-230.

[72] Deluzio, T. G. B., Seifu, D. G., Mequanint, K. (2011). 3D scaffolds in tissue engineering and regenerative medicine: beyond structural templates? Pharmaceut Biopro, 2011; 1: 267-281.

[73] Yu, Y., Moncal, K. K., Li, J., Peng, W., Rivero, I., Martin, J. A., Ozbolat, I. T. (2016). Three-dimensional bioprinting using self-assembling scalable scaffold-free tissue strands as a new biolink. Sci Rep, 2016; 6: 28714. (doi: 10.1038/srep28714)

[74] Jensen, G., Morrill, C., Huang, Y. (2018). 3D tissue engineering, an emerging technique for pharmaceutical research. Acta Pharmaceut. Sinica B, 2018; 8: 756-766.

[75] Duncker, H. R. (1971). The lung-air sac system of birds: a contribution to the functional anatomy of the respiratory apparatus. Ergeb Anat Entwicklungsgesch, 1971; 45: 1-171.

[76] Duncker, H. R. (1972). Structure of avian lungs. Respir Physiol, 1972; 14: 44-63.

[77] Duncker, H. R. (1974). Structure of avian respiratory tract. Respir Physiol, 1974; 22: 1-19.

[78] Scheid, P. (1979). Mechanisms of gas exchange in bird lungs. Rev Physiol Biochem Pharmacol, 1979; 86: 137-186.

[79] McLelland, J. (1989). Anatomy of the lungs and air sacs. In: Form and function in birds, vol. IV (King AS, McLelland J eds). London; Academic Press: 1989: pp. 221-279.

[80] Powell, F. L., Hopkins, S. R. (2004). Comparative physiology of lung complexity: implications for gas exchange. News Physiol Sci., 2004; 19: 55-60.

[81] Maina, J. N. (2005). The lung air sac system of birds: development, structure, and function. Heidelberg; Springer.

[82] Maina, J. N. (2006). Development, structure and function of a novel respiratory organ, the lung-air sac system of birds: To go where no other vertebrate has gone. Biol Rev., 2006; 81: 545-579.

[83] Maina, J. N. (2017). Pivotal debates and controversies on the structure and function of the avian respiratory system: setting the record straight. Biol Rev., 2017; 92: 1475-1504.

[84] Harvey, E. P., Ben-Tal, A. (2015). Robust unidirectional airflow through avian lungs: new insights from a piecewise linear mathematical mode. PLoS Comput Biol, 2015; 12(2): e1004637.

[85] Coitier, V. (1573). Anatomie avium. Externum et Internarum Praecipalium Humani Corporis Partium Tabulae atque Anatomicae Exercitationes Observationesque Varieae. Germany; Norimbergae: 1573; pp. 130-133.

[86] Maina, J. N. (2011). Bioengineering aspects in the design of gas exchangers: comparative evolutionary, morphological, functional, and molecular perspectives. Heidelberg: Springer.

[87] Fedde, R. (1980). The structure and gas flow pattern in the avian lung. Poult Sci., 1980; 59: 2642-2653.

[88] Wang, N., Banzett, R. B., Nations, C. S., Jenkins, E. A. (1992). An aerodynamic valve in the avian primary bronchus. J Exp Biol, 1992; 262: 441-445.

[89] Maina, J. N., Singh, P., Moss, E. A. (2009). Inspiratory aerodynamic valving occurs in the ostrich, Struthio camelus lung: computational fluid dynamics study under resting unsteady state inhalation. Respir Physiol Neurobiol, 2009; 169: 262-270.

[90] Maina, J. N. The Morphometry of the avian lung. In: Form and function in birds, vol. 4 (King AS, McLelland J eds). London; Academic Press: pp. 307-368.

[91] Maina, J. N., King, A. S., Settle, J. G. (1989). An allometric study of the pulmonary morphometric parameters in 
birds, with mammalian comparison. Philos Trans R Soc Lond B, 1989; 326: 1-57.

[92] Tucker, V. A. Energetics of natural avian flight. In: Avian energetics (Paynter RA ed). Cambridge (MA); Nuttal Ornithological Club: pp. 298-333.

[93] Maina, J. N. (2000). What it takes to fly: the novel respiratory structural and functional adaptations in birds and bats. J Exp Biol, 2000; 203: 3045-3064.

[94] Altshuler, D. L., Dudley, R. (2006). The physiology and biomechanics of avian flight at high altitude. Integr Comp Biol, 2006; 46: 4-8.

[95] Scott, G. R. (2011). Elevated performance: the unique physiology of birds that fly at high altitudes. J Exp Biol, 2011; 214: 2455-2462.

[96] Scott, G. R., Dawson, N. J. (2017). Flying high: The unique physiology of birds that fly at high altitudes. In: The biology of the avian respiratory system: evolution, development, structure and function (Maina JN ed). Heidelberg; Springer; 2017: pp. 113-128.

[97] Laguë, S. L. (2017). High altitude champions: birds that live and migrate at altitude. J Appl Physiol 2017; 123 : 942-950.

[98] Senner, N. R., Stager, M., Verhoeven, M. A., Cheviron, Z. A., Piersma, T., Bouten, W. (2018). High-altitude shorebird migration in the absence of topographical barriers: avoiding high air temperatures and searching for profitable winds. Proc. R. Soc. B, 2018; 285: 20180569.

[99] Rueden, C. T., Schindelin, J., Hiner, M. C., DeZonia, B. E., Walter, A. E., Arena, E. T., Eliceiri, K. W. (2017). ImageJ2: ImageJ for the next generation of scientific image data. BMC Bioinfornatics, 2017; 18: 529.

[100] Frank, J., Radermacher, M., Pencze, P., Zhu, J., Li, Y., Ladjadj, M., Leith, A. (1996). SPIDER and WEB: Processing and visualization of images in 3D electron microscopy and related fields. J Struct Biol, 1996; 116: 190-199.

[101] Maina, J. N., Woodward, J. D. (2009). Three-dimensional serial section computer reconstruction of the arrangement of the structural components of the parabronchus of the ostrich, Struthio camelus lung. Anat Rec., 2009; 292: 1685-1698.

[102] Rau, T. S., Hussong, A., Herzog, A., Majdani, O., Lenarz, T., Leinung, M. (2011). Accuracy of computer-aided geometric 3D reconstruction based on histological serial microgrinding preparation. Comput Methods Biomech Biomed Engineer, 2011; 14: 581-594.

[103] Pintilie, G. D., Zhang, J., Goddard, T. D., Chiu, W., Gossard, D. C. (2010). Quantitative analysis of cryo-EM density map segmentation by watershed and scale-space filtering, and fitting of structures by alignment to regions. J Struct Biol, 2010; 170: 427-38.

[104] Pattersen, E. F., Goddard, T. D., Huang, C. C., Couch, G. S., Greenblatt, D. M., Meng, E. C., Ferrin, T. E. (2004). UCSF Chimera-a visualization system for exploratory research and analysis. J Comput Chem, 2004; 5: 1605-1612.

[105] Abdalla, M. A. (1989). The blood supply to the lung. In: Form and function in birds, vol. 4 (King AS, McLelland J eds). San Diego; Academic Press; 1989: pp. 281-306.

[106] Abdalla, M. A., King, A. S. (1975). The functional anatomy of the pulmonary circulation of the domestic fowl. Respir Physiol, 1975; 23: 267-290.

[107] Abdalla, M. A., King, A. S. (1976). Pulmonary arteriovenous anastomoses in the avian lung: do they exist. Respir Physiol, 1976; 27: 187-191.

[108] Abdalla, M. A., King, A. S. (1976). The functional anatomy of the bronchial circulation of the domestic fowl. J Anat, 1976; 121: 537-550.

[109] Abdalla, M. A., King, A. S. (1977). The avian bronchial arteries: species variations. J Anat, 1977; 123: 697-704.

[110] King, A. S. (1966). Structural and functional aspects of the avian lung and its air sacs. Internat J Rev Gen Exp Zool, 1966; 2: 171-267.

[111] Maina, J. N. (1982). A scanning electron microscopic study of the air and blood capillaries of the lung of the domestic fowl (Gallus domesticus). Experientia, 1982; 35: 614-616.

[112] Maina, J. N. (1988). Scanning electron microscopic study of the spatial organization of the air- and the blood con- 
ducting components of the avian lung, Gallus gallus variant domesticus. Anat Rec., 1988; 222: 145-153.

[113] Makanya, A. N., Kavoi, B. M., Djonov, V. (2014). Three-dimensional structure and disposition of the air conducting and gas exchange conduits of the avian lung: the domestic duck (Cairina moschata). J Anat, 2014; 1: 1-9.

[114] West, N. H., Bamford, O. S., Jones, D. R. (1977). A scanning electron microscope study of the microvasculature of the avian lung. Cell Tissue Res, 1977; 176: 553-564.

[115] Makanya, A. N., Djonov, V. (2008). Development and spatial organization of the air conduits in the lung of the domestic fowl, Gallus variant domesticus. Microsc Res Tech, 2008; 71: 689-702.

[116] Makanya, A. N., Djonov, V. (2009). Parabronchial angioarchitecture in developing and adult chickens. J Appl Physiol, 2009; 106: 1959-69.

[117] Pandey, A. K., Praveen, P. K., Ganguly, S., Para, P. A., Wakchaure, R., Saroj, K., Mahajan, T. (2015). Avian respiratory and physiology with its interspecies variations: a review. World J Pharmacol Life Sci, 2015; 1: 137-148.

[118] Radu, C., Radu, L. (1971). Le dispositive vasculaire du poumon chez les oiseaux domestiques. Revue de Medecine Veterinaire, 1971; 122: 1219-1226.

[119] Weibel, E. R. (1984). The pathway for oxygen: structure and function in the mammalian respiratory system. Cambridge (MA); Harvard University Press: 1984.

[120] Schittny, J., Burri, P. H. (2003). Morphogenesis of the mammalian lung: aspects of structure and extracellular matrix. In: Lung development and regeneration (Massaro DJ, Massaro GC, Chambon P eds). New York; Marcel; 2003: pp. 275-316.

[121] Moura, R. S., Correia-Pinto, J. (2017). Molecular aspects of avian lung development. In: The biology of the avian respiratory system: evolution, development, structure and function (Maina JN ed). Berlin; Springer; 2017: pp. 129-146.

[122] Duncker, H. R. (1978). Development of the avian respiratory and circulatory systems. In: Respiratory function in birds, adult and embryonic (Duncker HR ed). Berlin; Springer; 1978: pp. 260-273.

[123] Maina, J. N. (2003). A systematic study of the development of the airway (bronchial) system of the avian lung from days 3 to 26 of embryogenesis: a transmission electron microscopic study of the domestic fowl, Gallus gallus variant domesticus. Tissue Cell, 2003; 35: 375-391.

[124] Maina, J. N. (2003). Developmental dynamics of the bronchial- (airway) and air sac systems of the avian respiratory system from days 3 to 26 of life: a scanning electron microscopic study of the domestic fowl, Gallus gallus variant domesticus. Anat Embryol, 2003; 207: 119-134.

[125] Burri, P. H. (1984). Fetal and postnatal development of the lung. Ann Rev Physiol, 1984; 46: 617-628.

[126] Burri, P. H. (1985). Development and growth of the human lung. In: Handbook of physiology, section 3: the respiratory system (Fishman AP, Fisher AB eds). Bethesda (MD); American Physiological Society; 1985: pp. 1-46.

[127] Hughes, L. C., Archer, C. W., Gwynn, I. (2005). The ultrastructure of mouse articular cartilage: collagen orientation and implications for tissue functionality. A polarised light and scanning electron microscope study and review. Europ Cells Materials, 2005; 9: 68-84.

[128] Woodward, J. D., Wepf, R., Sewell, B. T. (2009). Three-dimensional reconstruction of biological macromolecular complexes from in-lens scanning electronmicro-graphs. J Microsc, 2009; 234: 287-292.

[129] Peddie, C. J., Collinson, L. M. (2014). Exploring the third dimension: volume electron microscopy comes of age. Micron, 2014; 61: 9-19. 